\title{
Avaliação do teor de isoflavonas de "suplementos nutricionais à base de soja"
}

\author{
Maria Inés Genovese, Márcia da Silva Pinto, Ana Cristina Lopes Barbosa, Franco Maria Lajolo*
}

Departamento de Alimentos e Nutrição Experimental, Faculdade de Ciências Farmacêuticas, Universidade de São Paulo

*Correspondência:

F. M. Lajolo

Departamento de Alimentos e

Nutrição Experimental

Faculdade de Ciências Farmacêuticas

Universidade de São Paulo

Av. Prof. Lineu Prestes, 580. Bloco 14, 05508-900, São Paulo, SP, Brasil.

E-mail: fmlajolo@usp.br
Devido aos possiveis efeitos benéficos, diversos produtos à base de soja (gérmen, extrato), na forma de cápsulas ou comprimidos, surgiram recentemente no mercado como fontes de isoflavonas. Neste trabalho avaliaram-se o teor e o perfil de isoflavonas dos produtos comercializados em drogarias e farmácias locais (São Paulo), para verificar se estavam de acordo com o especificado pelos fabricantes. A determinação foi realizada através de cromatografia líquida de alta eficiência com detetor com arranjo de diodos, de acordo com Genovese \& Lajolo (2001b). Os resultados mostraram que os produtos à base de soja apresentam teor de isoflavonas muito abaixo do valor indicado nos rótulos, entre $27 \%$ e $86 \%$ a menos. Em relação à distribuição de isoflavonas, os produtos foram divididos em dois grupos: os que apresentam predominância de daidzeina e derivados e os que apresentam predominância de genisteina e derivados. Isso sugere a necessidade de padronização e maior controle de qualidade desses produtos, considerando o seu uso para mulheres na menopausa.
Unitermos:

- Soja

- Suplementos nutricionais

- Isoflavonas

\section{INTRODUÇÃO}

Diversos estudos epidemiológicos têm demonstrado menor incidência de câncer de mama, próstata e cólon nas populações da Ásia (Setchell, Cassidy, 1999; Tham et al., 1998), onde o consumo per capita de soja é 20 a 50 vezes maior que nas populações ocidentais (Anderson, Garner, 1997). O órgão norte-americano que regulamenta alimentos e medicamentos, FDA (Food and Drug Administration), aprovou, em 1999, um “claim" em rótulos de produtos alimentícios enriquecidos com proteína de soja, relacionando-os à redução de risco de doenças cardiovasculares. Tais efeitos positivos da soja parecem estar relacionados não apenas às suas proteínas, mas também a outras substâncias associadas a elas, como as isoflavonas (Crouse et al., 1999). A soja é uma fonte rica das isoflavonas daidzeína, genisteína e gliciteína, que estão presentes no grão na forma de derivados glicosilados (malonilglicosídeos e b-glicosídeos desesterificados), sendo que durante o processamento pode ocorrer a formação de agliconas e acetilglicosídeos (Figura 1) (Genovese, Lajolo, 2001a). Um estudo recente realizado com 156 homens e mulheres saudáveis norte-americanos mostrou redução dos níveis de colesterol total e lipoproteína de baixa densidade (LDL) provocada pela presença na dieta de $25 \mathrm{~g}$ de proteína isolada de soja por dia, sendo o efeito 
proporcional ao seu conteúdo de isoflavonas (Crouse et al., 1999). Além deste, estudos em cultura de células, modelos animais e alguns ensaios clínicos em humanos indicaram ação das isoflavonas também na prevenção e diminuição de certos tipos de câncer não relacionados a hormônios, benefícios no tratamento da osteoporose e alívio dos sintomas da menopausa (Setchell, Cassidy, 1999; Tham et al., 1998; Anderson, Garner, 1997).

Embora não tenham sido estabelecidos os mecanismos de ação, as relações dose-efeito, as variações de acordo com idade, raça e gênero, efeitos colaterais e/ou tóxicos, interações medicamentosas e efeitos da dieta, observou-se o aparecimento no mercado de um grande número de produtos à base de soja, na forma de cápsulas ou comprimidos, com indicações de diversos efeitos benéficos, desde alívio dos sintomas da menopausa e redução do colesterol, à prevenção de osteoporose, câncer, endometriose e mal de Alzheimer. Com a polêmica em torno dos hormônios sintéticos, o uso das isoflavonas como alternativa às terapias de reposição hormonal vem se popularizando e fórmulas manipuladas vêm sendo prescritas pela classe médica. No entanto, segundo a Sociedade Norteamericana de Menopausa (Greenwood et al., 2000), os dados da literatura existentes para avaliação dos efeitos das isoflavonas em câncer de mama, massa óssea e secura vaginal são inadequados. Estes seriam, também, inconclusivos em relação aos efeitos sobre humanos, questio- nando-se se são devidos às isoflavonas apenas ou às isoflavonas associadas a outros componentes dos alimentos. Posição similar foi adotada pela Sociedade Brasileira de Endocrinologia e Metabologia durante o $25^{\circ}$ Congresso Brasileiro de Endocrinologia, em Brasília, em setembro de 2002, onde se recomendou a não substituição das terapias hormonais convencionais pelo uso de fito-hormônios (www.sbem.org.br).

Embora nos Estados Unidos estes produtos sejam denominados "soy supplements", no Brasil a legislação não permite as designações "suplemento alimentar", "suplemento nutricional", "complemento alimentar" ou "complemento nutricional". Os produtos à base de soja encontrados no comércio não podem ser considerados alimentícios por apresentarem alegações medicamentosas ou terapêuticas. Desta forma, esses produtos são considerados irregulares pela ANVISA (Agência Nacional de Vigilância Sanitária) na área de alimentos. Existem apenas dois produtos com isoflavonas registrados na ANVISA, como medicamentos fitoterápicos, sendo que apenas as indicações para alívio das ondas de calor associadas à menopausa e como auxiliar na redução dos níveis de colesterol são permitidas (www.anvisa.gov.br). Além de sua irregularidade, não há uma padronização das fontes de isoflavonas nem controle sobre os teores de isoflavonas e sua variação para a maioria dos produtos encontrados no comércio.

\section{Agliconas:}

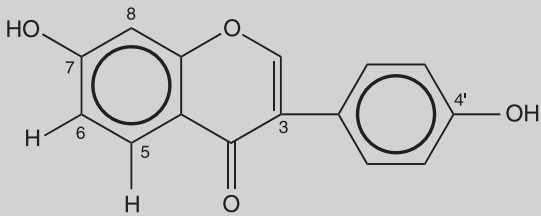

Daidzeína

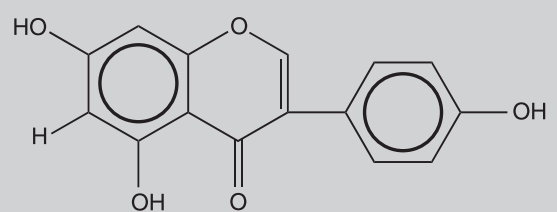

Genisteína

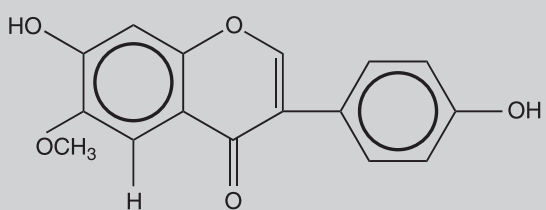

Gliciteína
Glicosídeos:

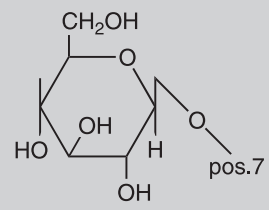

Acetil-isoflavonas:

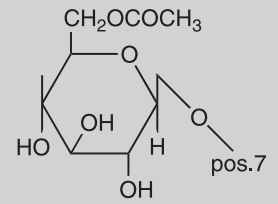

Malonil-isoflavonas:

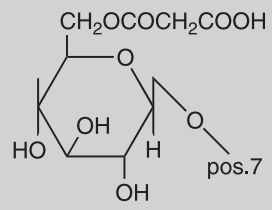

FIGURA 1 - Estrutura química das isoflavonas da soja. 
O objetivo deste trabalho foi determinar o teor de isoflavonas dos produtos à base de soja disponíveis em drogarias e farmácias de manipulação da cidade de São Paulo e avaliar se estes apresentam a quantidade especificada na embalagem. Objetivou-se, também, analisar a possível variação em relação à composição de isoflavonas, decorrentes do uso de diferentes fontes e/ou condições de processamento.

\section{MATERIAIS E MÉTODOS}

\section{Materiais}

Os produtos comercializados como fontes de isoflavonas foram obtidos em drogarias e farmácias de manipulação locais (São Paulo, SP) durante o ano de 2002, totalizando 12 amostras. Foram analisados: (1) Natu-soy Terra Verde ${ }^{\circledR}$ (Sanibras Bionutrientes Ltda., Pinhais, PR), (2) Natural Isoflavones ${ }^{\circledR}$ e (3) Bioflavona (Phytomare ${ }^{\circledR}, \mathrm{Ad}$ Oceanum Ind. e Com. Ltda., Gde. Florianópolis, SC), (4) Isoflavona Nutramed ${ }^{\circledR}$ (Braskap Ind. e Com. S/A, Sorocaba, SP), (5) Iso soy (Herborisa Prod. e Benef. de ervas Ltda., Londrina, PR), (6) Soy 50 Menopause Supplement ${ }^{\circledR}$ (Nature made Nutritional Products, E.U.A.), (7) Isoflavona + cálcio (Lab. Farmacêutico Vitamed Ltda., Caxias do Sul, RS), e (8) Isoflavine ${ }^{\circledR}$ (Herbarium Lab. Botânico Ltda., Colombo, PR). Apenas os produtos (6) e (7) são comercializados na forma de comprimidos, sendo os demais na forma de cápsulas. Analisou-se, também, a matéria-prima utilizada pelas farmácias de manipulação para o preparo das cápsulas de isoflavonas. A matéria-prima (9), comercializada pela Galena Química e Farmacêutica Ltda. (Campinas, SP), apresenta o nome comercial Isoflavin Beta ${ }^{\circledR}$, com conteúdo mínimo de $40 \%$ de isoflavonas, segundo o fabricante, e o país de origem é a China. Três amostras $(10,11$, e 12$)$ de cápsulas de isoflavonas ( 25 e $50 \mathrm{mg}$ ), de três farmácias de manipulação da região do Butantã, foram analisadas. Os produtos analisados (13) Sanavita ${ }^{\circledR}$ Café da manhã e (14) Refeições salgadas (Sanavita Alimentos Funcionais Ltda., Piracicaba, SP) também foram adquiridos no comércio local. Todos os reagentes e solventes utilizados foram de grau analítico (metanol, ácido acético) ou para cromatografia líquida (metanol, acetonitrila).

\section{Métodos}

\section{Extração das isoflavonas}

Dez cápsulas de cada produto tiveram seu conteúdo retirado, homogeneizado e pesado. Os comprimidos (10 de cada amostra) foram previamente pulverizados em graal. A extração das isoflavonas a partir dos pós obtidos foi realizada com agitador mecânico e barra magnética por 2 horas a $4{ }^{\circ} \mathrm{C}$, na proporção de 1:20 (m/v), com metanol $80 \%$ (Genovese, Lajolo, 2001b). Os extratos foram filtrados utilizando-se papel de filtro Whatman $\mathrm{n}^{\circ} 6$ para balão volumétrico, onde tiveram seu volume completado com água destilada para $250 \mathrm{~mL}$, para posterior aplicação em colunas de extração em fase sólida. As extrações foram realizadas em triplicata.

\section{Extração em fase sólida}

A extração em fase sólida de isoflavonas foi realizada utilizando-se colunas de $1 \mathrm{~g}$ de poliamida (CC 6, Macherey-Nagel GmbH \& Co., Alemanha) em seringas próprias de $6 \mathrm{~mL}$, da HPLC Technology Co. Ltd. (Hertfordshire, Reino Unido). As colunas foram pré-condicionadas pela passagem de $20 \mathrm{~mL}$ de metanol e $40 \mathrm{~mL}$ de água. Alíquotas $(2,5 ; 5$ e $10 \mathrm{~mL})$ dos extratos obtidos anteriormente foram aplicadas diretamente nas colunas. Após a passagem das amostras, as colunas foram lavadas com $20 \mathrm{~mL}$ de água e a eluição das isoflavonas foi feita com 50 mL de metanol:amônia $(99,5: 0,5)$. Utilizou-se manifold Visiprep 24 DL da Supelco (Bellefonte, E.U.A.). Os eluatos foram submetidos a rotaevaporação (Rotavaporâ RE 120 - Büchi, Flawil, Suíça) até atingir o volume de $0,2 \mathrm{~mL}$, e a seguir completou-se o volume para $2 \mathrm{~mL}$ com metanol (grau HPLC). As amostras foram filtradas utilizando-se filtros de polietileno com membrana PTFE (Millipore Ltd., Bedford, E.U.A.) de 0,22 m de poro (Genovese, Lajolo, 2001b).

\section{Cromatografia líquida de alta eficiência}

A separação das isoflavonas foi realizada em coluna C 18 mBondapak (Waters, Milford, E.U.A.) de acordo com o método de Song et al. (1998). O cromatógrafo líquido utilizado foi o da Hewlett Packard (Palo Alto, E.U.A.) série 1100, equipado com injetor automático de amostras, bomba quaternária e detetor com arranjo de diodos, controlados pelo software ChemStation. Os padrões de daidzeína e genisteína foram obtidos da Sigma Chemicals Co. (St. Louis, E.U.A.), daidzina e genistina foram obtidos da Apin Chemicals Ltd. (Abingdon, Reino Unido), glicitina e gliciteína da Fujicco Co. Ltd. (Kyoto, Japão). A identificação foi feita a partir dos tempos de retenção e dos espectros. As amostras foram injetadas em duplicata. Os resultados foram expressos na forma de média \pm desviopadrão, como mg de isoflavonas por $100 \mathrm{~g}$ de amostra (b.u.), após normalização das diferenças de peso molecular das formas glicosiladas, por multiplicação da massa de cada derivado pela razão entre o peso molecular da respectiva aglicona e o peso molecular da forma glicosilada, conforme Song et al. (1998). 


\section{RESULTADOS E DISCUSSÃO}

Os produtos à base de soja analisados diferem quanto à fonte das isoflavonas. Como pode ser visto na Tabela I, as fontes de isoflavonas mais comuns são o gérmen de soja ou o extrato concentrado de soja. O produto (3) Bioflavona constitui-se de gérmen de soja enriquecido com isoflavonas e apresenta, portanto, concentração maior destas, de acordo com o fabricante. Já o produto (7) Isoflavona + Cálcio não traz nenhuma informação sobre a origem das isoflavonas. O produto (8) Isoflavine contém extrato de isoflavonas a $40 \%$, além de óleo de soja, provavelmente a mesma matéria-prima, das cápsulas preparadas nas farmácias de manipulação. Essas diversas origens das isoflavonas são importantes de serem consideradas, já que delas vai depender o conteúdo e perfil de isoflavonas presentes nos produtos.

TABELA I - Fontes de isoflavonas dos produtos comerciais

\begin{tabular}{ll}
\hline Produto & Fonte de isoflavonas \\
\hline (1) Natu-Soy & Gérmen de soja \\
(2) Natural Isoflavones & Gérmen de soja \\
(3) Bioflavona & Gérmen de soja enriquecido \\
(4) Isoflavona & Extrato de soja \\
(5) IsoSoy & Gérmen de soja \\
(6) Soy 50 Menopause & Extrato de soja \\
Supplement & \\
(7) Isoflavona + Cálcio & ? \\
(8) Isoflavine & Extrato de isoflavonas de \\
& soja a 40\% \\
\hline
\end{tabular}

? não informada
As isoflavonas se concentram no hipocótilo (erroneamente denominado gérmen) da semente de soja, sendo encontradas quantidades até dez vezes maiores que no cotilédone, e cerca de cem vezes maiores que na casca. No entanto, como o cotilédone corresponde a $90 \%$ da semente e o hipocótilo a apenas $2 \%$, a maior contribuição em termos de isoflavonas para o grão é do cotilédone, o que justifica a utilização de extratos concentrados obtidos a partir do grão de soja como fonte de isoflavonas. A utilização do gérmen ou de extrato concentrado de soja resulta em produtos com diferenças em sua composição em relação ao perfil das isoflavonas, já que no hipocótilo encontramse basicamente daidzina e glicitina, enquanto que no cotilédone há 20 vezes mais genistina que no hipocótilo (Eldridge, Kwolek, 1983).

Dos oito produtos comerciais analisados, seis são na forma de cápsulas e dois na forma de comprimidos. O conteúdo de isoflavonas, segundo os fabricantes, varia entre 13,5 e $60 \mathrm{mg}$ de isoflavonas por cápsula/comprimido e a recomendação de ingestão, entre 20 e $100 \mathrm{mg}$ de isoflavonas por dia (Tabela II). As indicações de efeitos benéficos, encontradas nas embalagens e/ou pela internet, no site dos fabricantes, vão desde ação antioxidante, prevenção e alívio dos sintomas da menopausa, osteopenia e osteoporose, diminuição do colesterol, redução do risco de doenças cardiovasculares, prevenção do aparecimento de câncer (mama e útero), endometriose e mal de Alzheimer. A matéria-prima (9) utilizada pelas farmácias para manipulação de cápsulas de isoflavonas vem acompanhada de recomendação de ingestão diária de 40 a $150 \mathrm{mg}$ de isoflavonas (Tabela II).

Os produtos da Sanavita ${ }^{\circledR}$ (13) Café da manhã e (14) Refeições salgadas, embora sejam classificados como alimentos e não como fitoterápicos, aparecem neste trabalho

TABELA II - Conteúdo e recomendações de isoflavonas dos produtos comerciais de acordo com os rótulos/embalagens

\begin{tabular}{lccc}
\hline Produto & $\begin{array}{c}\text { Isoflavonas } \\
\text { (mg/cápsula) }\end{array}$ & $\begin{array}{c}\text { Recomendação* } \\
\text { (cápsulas/dia) }\end{array}$ & $\begin{array}{c}\text { Total recomendado* } \\
\text { (mg isoflavona/dia) }\end{array}$ \\
\hline (1) Natu-Soy & $13,5-15$ & $3-4$ & $40,5-60$ \\
(2) Natural Isoflavones & 15 & $3-4$ & $45-60$ \\
(3) Bioflavona & 60 & 1 & 60 \\
(4) Isoflavona & 20 & 2 & 40 \\
(5) IsoSoy & 15 & $3-4$ & $45-60$ \\
(6) Soy 50 Menopause Supplement & $50^{* *}$ & $1-2^{* *}$ & $50-100$ \\
(7) Isoflavona + Cálcio & $20^{* *}$ & $1-2^{* *}$ & $20-40$ \\
(8) Isoflavine & 30 & 2 & 60 \\
(9) Isoflavin Beta & Pó & $100-375 \mathrm{mg}$ & 40 a 150 \\
\hline
\end{tabular}

*segundo o fabricante; ** comprimido 
por apresentarem, no folheto explicativo que os acompanha, indicações para redução dos sintomas da menopausa e dos riscos de osteoporose e certos tipos de câncer (mama e útero). Segundo o folheto, a ingestão de $120 \mathrm{~g}$ do produto por dia ( $80 \mathrm{~g}$ de Sanavita refeições salgadas +40 $\mathrm{g}$ de Sanavita café da manhã) corresponderia à ingestão de $64 \mathrm{mg}$ de isoflavonas.

A Tabela III mostra que nem os produtos comerciais (1 a 8), nem a matéria-prima (9), nem as fórmulas de manipulação (10 a 12), e nem os produtos Sanavita (13 e 14) apresentaram as quantidades de isoflavonas especificadas nas embalagens/rótulos. As diferenças encontradas para os produtos comerciais variaram entre - $27 \%$ (produto 2 , Natural Isoflavones) e - $86 \%$ (produto 4 , Isoflavona) em relação ao conteúdo de isoflavonas declarado. Para as três fórmulas $(10,11$ e 12) adquiridas em farmácias de manipulação esses valores foram de - 54 a - 66\%.

O menor conteúdo de isoflavonas encontrado nas cápsulas de manipulação parece estar de acordo com o menor conteúdo encontrado na matéria-prima (9) Isoflavin Beta analisada, em relação ao declarado (- $71 \%)$. O mesmo poderia ser aplicado para o produto (8) Isoflavine (- 57\%), que também leva em sua composição extrato de isoflavonas a $40 \%$. As cápsulas produzidas com esta matéria-prima apresentarão valores abaixo do especificado no rótulo, já que as farmácias e a indústria utilizarão o valor dado pelo fabricante para o cálculo do conteúdo de isoflavonas presentes no produto final.
Já os produtos Sanavita (13 e 14) não apresentaram quantidades detectáveis de isoflavonas, que seriam provenientes da soja presente em sua composição. No entanto, além desta, os produtos apresentam também aveia, gérmen de trigo, castanha de caju e gergelim, o que significa que o conteúdo de isoflavonas do produto dependerá da proporção em que a soja está presente e do conteúdo de isoflavonas da mesma, que é altamente variável.

$\mathrm{O}$ teor de isoflavonas da soja difere de acordo com a variedade e com as condições climáticas e do solo. A análise de 210 cultivares de soja plantados em Dakota do Sul (E.U.A.) mostrou teores de isoflavonas entre 116 e 274 mg por $100 \mathrm{~g}$ (Wang et al., 2000), e para 15 diferentes cultivares de soja do Paraná entre 54 e 147 mg por 100 g, sendo que a concentração média era $31 \%$ maior para as cultivares de Ponta Grossa (120 mg/100 g) em comparação com as de Londrina $(82 \mathrm{mg} / 100 \mathrm{~g})$. Essas diferenças foram atribuídas a diferenças de temperatura e solo entre as duas regiões (Carrão-Panizzi et al., 1998). Eldridge e Kwolek (1983) também encontraram variação, entre 46 e $195 \mathrm{mg}$ por $100 \mathrm{~g}$, para a mesma variedade cultivada em diferentes regiões. Ainda, a mesma variedade cultivada na mesma região apresentou variação no conteúdo de isoflavonas de um ano para o outro, entre 118 e $331 \mathrm{mg}$ isoflavonas por $100 \mathrm{~g}$ (Wang, Murphy, 1994a), o que foi atribuído a fatores climáticos e ambientais.

Essas variações dos teores de isoflavonas da soja mostram a inadequação da utilização de valores médios

TABELA III - Conteúdo de isoflavonas dos produtos comerciais e dos produtos Sanavita

Isoflavonas (mg/cápsula)

\begin{tabular}{lccc} 
Produto & Rótulo & Encontrado & Variação $(\%) * *$ \\
\hline (1) Natu-Soy & $13,5-15$ & 2,8 & -79 \\
(2) Natural Isoflavones & 15 & 11,0 & -27 \\
(3) Bioflavona & 60 & 21,0 & -65 \\
(4) Isoflavona & 20 & 2,7 & -86 \\
(5) IsoSoy & 15 & 4,2 & -72 \\
(6) Soy 50 Menopause Supplement & $50 *$ & 22,5 & -55 \\
(7) Isoflavona + Cálcio & $20 *$ & -82 \\
(8) Isoflavine & 30 & 12,9 & -57 \\
(9) Isoflavin Beta & $42,6 \mathrm{mg} / 100 \mathrm{~g}$ & $12,4 \mathrm{mg} / 100 \mathrm{~g}$ & -71 \\
(10) Farmácia de manipulação 1 & 25 & 11,5 & -54 \\
(11) Farmácia de manipulação 2 & 50 & 16,9 & -66 \\
(12) Farmácia de manipulação 3 & 50 & 21,7 & -57 \\
(13) Sanavita - Café da Manhã (CM) & $64 \mathrm{mg} / 120 \mathrm{~g}$ & $\mathrm{n} . \mathrm{d}$. & -100 \\
(14) Sanavita - Refeições Salgadas (RS) & $(80 \mathrm{~g} \mathrm{RS} \mathrm{+} \mathrm{40} \mathrm{g} \mathrm{CM)}$ & & -100
\end{tabular}

*comprimido; **em relação ao indicado no rótulo; n.d. - não detectado. 
obtidos para a soja para o cálculo do teor de isoflavonas do produto final. Além disso, em nenhum dos rótulos dos produtos analisados está especificada a forma de se expressar o conteúdo total de isoflavonas, ou seja, se a massa total foi calculada a partir da soma da massa dos derivados glicosilados mais os derivados glicosilados e esterificados mais as agliconas, ou se refere a resultados normalizados para massa equivalente das respectivas agliconas. Resultados expressos na forma de derivados glicosilados superestimariam os teores de isoflavonas já que o seu peso molecular é quase o dobro do das agliconas. Como as formas que são absorvidas são as agliconas, o recomendável para expressar o conteúdo de isoflavonas dos produtos comerciais é normalizar os resultados para massa das agliconas e/ou apresentar as quantidades molares das isoflavonas (Song et al., 1998; Nurmi et al., 2002). Os valores expressos no presente trabalho são em massa equivalente de agliconas. Sem esta normalização, o teor de isoflavonas encontrado para o produto (2) Natural Isoflavones, de $16,8 \pm 0,3 \mathrm{mg} /$ cápsula, representa $12 \%$ a mais do especificado no rótulo. Para os outros produtos, no entanto, os resultados mesmo expressos dessa forma continuam abaixo do declarado, entre 30 a $84 \%$ a menos, respectivamente para (6) Soy 50 Supplement e (4) Isoflavona.

Nurmi et al. (2002) analisaram quinze suplementos à base de soja comercializados na Finlândia e, dos onze que especificavam o conteúdo de isoflavonas, apenas um estava de acordo com o rótulo. Os outros dez produtos apresentaram valores entre 23 e $69 \%$ menores. Setchell et al. (2001) compararam os resultados obtidos, sem conversão para agliconas, com o teor declarado de 30 suplementos e observaram que 24 deles estavam abaixo do indicado.

Em relação ao perfil de isoflavonas dos produtos, a Tabela IV mostra que este também variou significativamente. Os produtos contendo gérmen de soja (1) NatuSoy, (2) Natural Isoflavones e (5) IsoSoy apresentaram um baixo conteúdo de agliconas, de 4 a $8 \%$, enquanto os demais apresentaram, em sua maioria, mais de $40 \%$ de agliconas. O produto (6) Soy 50 Menopause Supplement, embora contenha extrato de soja, apresentou perfil similar ao do (2) Natural Isoflavones, caracterizado pelo alto conteúdo de $\beta$-glicosídeos. Já a matéria-prima (9) Isoflavin Beta, os produtos manipulados $(10,11,12),(8)$ Isoflavine (extrato a $40 \%$, provavelmente o mesmo ou similar ao Isoflavin Beta), e (3) Bioflavona (gérmen enriquecido, provavelmente também com extrato concentrado) apresentaram perfil similar, constituído por cerca de $50 \%$ de agliconas e $50 \%$ de $\beta$-glicosídeos.

Os teores de malonilderivados são altamente variáveis já que estes são extremamente instáveis ao calor. Normalmente há a desesterificação com formação dos respectivos $\beta$-glicosídeos, porém em condições de processamento associadas a calor seco ou baixos teores de umidade, como a tostagem da farinha ou a extrusão de derivados protéicos, ocorre a formação de acetiglicosídeos. Já as agliconas são formadas pela ação das $\beta$-glicosidades da própria soja ou fúngicas. Além das alterações provocadas pelo processamento, Wang e Murphy (1994a) observaram também diferenças no perfil de isoflavonas entre as variedades de soja americanas e as japonesas, as últimas com maior teor de malonilglicitina e também maiores propor-

TABELA IV - Perfil de isoflavonas dos produtos comerciais

\begin{tabular}{lcccc}
\hline & & \multicolumn{2}{c}{$\%$} & \\
Produto & $\boldsymbol{\beta}$-glicosídeos & M-glicosídeos & A-glicosídeos & agliconas \\
\hline (1) Natu-Soy & $34,9 \pm 0,5$ & $58,6 \pm 0,4$ & $2,30 \pm 0,02$ & $4,3 \pm 0,1$ \\
(2) Natural Isoflavones & $82,9 \pm 0,1$ & $9,4 \pm 0,2$ & n.d. & $7,7 \pm 0,1$ \\
(3) Bioflavona & $52,8 \pm 0,8$ & n.d. & n.d. & $47,2 \pm 0,8$ \\
(4) Isoflavona & $55,2 \pm 0,2$ & n.d. & $4,2 \pm 0,1$ & $40,6 \pm 0,1$ \\
(5) IsoSoy & $52,7 \pm 0,2$ & $31,3 \pm 0,1$ & $11,2 \pm 0,1$ & $4,8 \pm 0,1$ \\
(6) Soy 50 Menopause Supplement & $89,6 \pm 0,2$ & n.d. & n.d. & $10,4 \pm 0,2$ \\
(7) Isoflavona + Cálcio & $30 \pm 1$ & $37,6 \pm 0,7$ & n.d. & $32,0 \pm 0,3$ \\
(8) Isoflavine & $45 \pm 1$ & n.d. & $1,5 \pm 0,1$ & $53 \pm 1$ \\
(9) Isoflavin Beta & $54 \pm 3$ & n.d. & n.d. & $46 \pm 3$ \\
(10) Farmácia de manipulação 1 & $54,5 \pm 0,1$ & n.d. & n.d. & $45,5 \pm 0,1$ \\
(11) Farmácia de manipulação 2 & $50 \pm 1$ & n.d. & n.d. & $50 \pm 1$ \\
(12) Farmácia de manipulação 3 & $38 \pm 2$ & n.d. & n.d. & $62 \pm 2$ \\
\hline
\end{tabular}

M-glicosídeos: malonilglicosídeos; A-glicosídeos: acetilglicosídeos. n.d. não detectado. 
ções entre malonilgenistina e genistina, e entre malonildaidzina e daidzina. Não há dados sobre a biodisponibilidade dessas diferentes formas, mas um estudo recente demonstrou ser a absorção das agliconas, em humanos, mais rápida e eficiente que a dos glicosídeos (Izumi et al., 2000).

A distribuição das isoflavonas presentes nos produtos comerciais em total de daidzeína, genisteína e gliciteína (agliconas naturalmente presentes mais os derivados glicosilados) é apresentada na Tabela V. De acordo com sua distribuição, os produtos podem ser divididos em dois grandes grupos: os que contêm predominância de genisteína e seus derivados e os que contêm predominância de daidzeína e seus derivados. O grupo que contém predominância de daidzeína apresentou porcentagens de gliciteína $\geq 25 \%$ e o que contém predominância de genisteína, porcentagens de gliciteína $\leq 10 \%$. A composição do primeiro grupo é característica de produtos à base de gérmen de soja e a do segundo grupo corresponde a produtos contendo extrato de soja como fonte de isoflavonas. Estes resultados permitiram determinar que o produto (7) Isoflavona + Cálcio, que não apresenta seus ingredientes no rótulo, é feito à base de gérmen de soja.

Essas diferenças na composição de isoflavonas poderiam resultar em diferenças na sua ação no organismo, já que a atividade estrogênica da gliciteína é três vezes maior que a da genisteína (Song et al., 1999). Desta forma, enquanto não estiverem bem estabelecidos os efeitos biológicos das isoflavonas, não se pode assegurar os mesmos resultados para produtos diferentes.
Os resultados obtidos indicam maior necessidade de controle da composição desses produtos e também cuidado na indicação dos mesmos para as mulheres na menopausa. Esse último fato é ainda ressaltado pela falta de estudos que comprovem definitivamente que a ingestão de isoflavonas está relacionada aos diversos efeitos preventivos apregoados e mesmo com relação apenas com o alívio nos sintomas da menopausa.

\section{CONCLUSÕES}

Todos os produtos analisados apresentaram conteúdos totais de isoflavonas em desacordo com o apresentado nos rótulos/embalagens. Torna-se, portanto, necessário o controle efetivo desses produtos, devendo-se, ainda, ressaltar a necessidade de padronização da metodologia de análise e da forma de expressar os teores de isoflavonas, que seria preferencialmente na forma de agliconas.

\section{ABSTRACT}

\section{Isoflavone content of soy dietary supplements}

Because of the possible health effects, a large number of soy isoflavone products are now available on the Brazilian market. Our objective was to determine the isoflavone content and distribution in soy dietary supplements commercialized in Brazil and verify the accordance to the isoflavone content supplied by the producers. Determinations were made by high performance liquid

TABELA V - Distribuição de isoflavonas nos produtos comerciais

Produto

(1) Natu-Soy

(2) Natural

(3) Bioflavona

(4) Isoflavona

(5) IsoSoy

(6) Soy 50 Menopause Supplement

(7) Isoflavona + Cálcio

(8) Isoflavine

(9) Isoflavin Beta

(10) Farmácia de manipulação 1

(11) Farmácia de manipulação 2

(12) Farmácia de manipulação 3
$\%$ *

Daidzeína total

Genisteína total

Gliciteína total

$$
\begin{aligned}
52,9 & \pm 2,2 \\
60,3 & \pm 0,4 \\
39,6 & \pm 0,7 \\
31,9 & \pm 2,2 \\
46,9 & \pm 2,2 \\
39,05 & \pm 0,04 \\
52,1 & \pm 0,5 \\
29,7 & \pm 0,5 \\
37 & \pm 2 \\
36,66 & \pm 0,02 \\
36,8 & \pm 1,1 \\
31 & \pm 2
\end{aligned}
$$

$31,1 \pm 0,7$

$25,4 \pm 0,4$

$8,4 \pm 0,5$

$7,0 \pm 0,7$

$37,8 \pm 0,7$

$13,4 \pm 0,1$

$29,5 \pm 0,3$

$5,7 \pm 0,1$

$10,6 \pm 0,8$

$8,83 \pm 0,02$

$7,3 \pm 0,2$

$7,2 \pm 0,6$

* porcentagem de cada isoflavona representada pela soma das formas aglicona, $\beta$-glicosídeo desesterificado, malonil e acetilglicosídeo. 
chromatography and photodiode array detection of the intact isoflavones without acid hydrolysis. Total isoflavone content varied significantly among products, from 3 to 22 $\mathrm{mg} / 100 \mathrm{~g}$ (expressed as aglycones) and was much lower than the values found in the product labels (27 to 86\% less). Isoflavone distribution varied according to isoflavone source and products could be classified in two groups, those having daidzein predominance and those having genistein predominance. Based on these data, we conclude that there is a need of better standardization and quality control of these products and also a clear regulation.

UNITERMS: Soy dietary supplements. Isoflavones.

\section{REFERÊNCIAS BIBLIOGRÁFICAS}

ANDERSON, J. J. B.; GARNER, S. C. The effects of phytoestrogens on bone. Nutr. Res., v. 17, p. 1617-1632, 1997.

ANVISA. Agência Nacional de Vigilância Sanitária. Workshop sobre isoflavonas realizado em 29 de agosto de 2002. Disponível em: http://www.anvisa.gov.br/faq/ al_isoflavonas.htm. Acesso em 10 de outubro de 2002.

CARRAO-PANIZZI, M. C.; KITAMURA, K.; BELEIA, A. D.; OLIVEIRA, M. C. N. Influence of growth locations on isoflavone contents in Brazilian soybeans cultivars. Breeding Sci., v. 48, p. 409-413, 1998.

CROUSE, J. R. $3^{\text {rd }}$; MORGAN, T.; TERRY, J.G.; ELLIS, J.; VITOLINS, M.; BURKE, G. L. A randomized trial comparing the effect of casein with that of soy protein containing varying amounts of isoflavones on plasma concentration of lipids and lipoproteins. Arch. Intern. Med., v. 159, p. 2070-2076, 1999.

ELDRIDGE, A. C.; KWOLEK, W. F. Soybean Isoflavones: effects of environment and variety on composition. $J$. Agric. Food Chem., v. 31, p. 394-396, 1983.

GENOVESE, M. I.; LAJOLO, F. M. Isoflavonas da soja: fatores que influem nos tipos e teores em alimentos. Food Ingred., v. 11, p. 62-64, $2001 \mathrm{a}$.

GENOVESE, M. I.; LAJOLO, F. M. Determinação de isoflavonas em derivados de soja. Ciênc. Tecnol. Alim., v. 21, p. $86-93,2001 b$.
GREENWOOD, S.; BARNES, S.; CLARKSON, T. B.; EDEN, J.; HELFERICH, W. G.; HUGHES, C.; MESSINA, M.; SETCHELL, K. D. R. The role of isoflavones in menopausal health: Consensus opinion of the North American Menopause Society. Menopause, v. 7, p. 215-229, 2000 .

IZUMI, T.; PISKULA, M. K.; OSAWA, S.; OBATA, A.; TOBE, K.; SAITO, M.; KATAOKA, S.; KUBOTA, Y.; KIKUCHI, M. Soy isoflavone aglycones are absorbed faster and in higher amounts than their glucosides in humans. J. Nutr., v. 130, p. 1695-1699, 2000.

NURMI, T.; MAZUR, W.; HEINOMEN, S.; HOKKONEN, J.; ADLERCREUTZ, H. Isoflavone content of the soy based supplements. J. Pharm. Biomed. Anal., v. 28, p. 1$11,2002$.

SBEM. Sociedade Brasileira de Endocrinologia e Metabologia. Folha da Sociedade Brasileira de Endocrinologia e Metabologia on line, edição ${ }^{\circ} 01$ do $25^{\circ} \mathrm{CBEM}$, dezembro de 2002. Disponível em: http:// www.sbem.org.br/folha2/fsbem.htm. Acesso em 19 de novembro de 2002.

SETCHELL, K. D. R.; CASSIDY, A. Dietary isoflavones: biological effects and relevance to human health. J. Nutr., v. 129, p. 758S-767S, 1999.

SETCHELL, K. D. R.; BROWN, N. M.; DESAI, P.; ZIMMER-NECHEMIAS, L.; WOLFE, B. E.; BRASHEAR, W. T.; KIRSCHNER, A. S.; CASSIDY, A. Bioavailability of pure isoflavones in healthy humans and analysis of commercial soy isoflavone supplements. J. Nutr., v. 131, p. 1362S-1375S, 2001.

SONG, T.; BARUA, K.; BUSEMAN, G.; MURPHY, P. A. Soy isoflavones analysis: quality control and a new internal standard. Am. J. Clin. Nutr., v. 68, p. 1474S1479S, 1998.

SONG, T. T.; HENDRICH, S.; MURPHY, P. A. Estrogenic Activity of Glycitein a Soy Isoflavone. J. Agric. Food Chem., v. 47, p. 1607-1610, 1999.

THAM, D. M.; GARDNER, C. D.; HASKELL, W. L. Clinical review 97: Potential health benefits of dietary phytoestrogens. J. Clin. Endocrin. Metab., v. 83, p. 2223 $2235,1998$. 
WANG, C.; SHERRARD, M.; PAGADALA, S.; WIXON, R.; SCOTT, R. A. Isoflavone content among maturity group 0 to II soybeans. J. Am. Oil Chem. Soc., v. 77, p. 483-487, 2000.
WANG, H.; MURPHY, P. A. Isoflavone composition of American and Japonese soybean in Iowa: effects of variety, crop year, and location. J. Agric. Food Chem. v. 42, p. 1674-1677, 1994a.

Recebido para publicação em 05 de dezembro de 2002. 